\title{
Incidence of recurrent stroke in primary care during preventive treatment based on perindopril with or without indapamide
}

\author{
M. V. Padma, Subhash Kaul* \\ Department of Neurology, All India Institute of Medical Sciences, New Delhi, *The Nizam's Institute of Medical Sciences, Hyderabad, \\ India
}

Background: Although the risk reduction of recurrent stroke with angiotensin converting enzyme-inhibitors with or without a diuretic has been demonstrated under randomized double blind conditions of the PROGRESS study, it is unclear whether the benefit is reflected in primary care practice and in populations with different demographic and clinical characteristics. Aim: To assess the effect and acceptability of perindopril with or without indapamide prevention of recurrent stroke, as reflected by its incidence. Setting and Design: Multicentre, prospective, observational study in the setting of primary care throughout India. Materials and Methods: Patients with a stable stroke or transient ischaemic attack (TIA) received a 12-month perindopril \pm indapamide-based regimen, similar to that used in PROGRESS. The principal outcome was the annual incidence of recurrent stroke. Statistical Analysis: Summary statistics and the Kaplan-Meier procedure. Results: The mean age of 298 patients was 58.3 years $(\mathrm{SD}=12.6) .229$ (77.5\%) had an ischaemic stroke; 231 (77.5\%) were hypertensive; 200 (85.5\%) were receiving aspirin and $81(27.2 \%)$ statins. During the 12-month perindopril-based treatment, there were $8(2.7 \%)$ recurrent strokes, with a Kaplan-Meier estimate of strokes plus TIA of $3.3 \%(95 \% \mathrm{Cl}, 1.0-5.6)$. Conclusions: The incidence of recurrent stroke is similar to that observed under double blind randomized conditions in the treatment arm of the PROGRESS study. This suggests that perindopril \pm indapamide-based prevention may be effective in reducing risk of recurrent stroke, (although the uncontrolled study design does not actually demonstrate this), in the setting of day-to-day clinical practice and among patients with different demographic and clinical characteristics than the PROGRESS population.

Key words: Cerebrovascular accident, perindopril, recurrent stroke

\section{Introduction}

Of the 20 million people worldwide who suffer a stroke each year, about half either die or are disabled. ${ }^{[1,2]}$ Surveys suggest that the prevalence of stroke in India is about $0.5 \%$, with an annual mortality of over $1,00,000$, that represents $1.2 \%$ of all deaths. ${ }^{[3]}$ In spite of enormous advances in the understanding and treatment of stroke, prevention remains the strategy of paramount importance. ${ }^{[4]}$

Effective control of hypertension has the potential to reduce the risk of primary stroke by nearly $40 \%$, but unfortunately half of all hypertensive patients receive no treatment and of those who do, not more than half are adequately controlled. ${ }^{[5]}$ Since the 5-year risk of a second stroke or transient ischaemic attack (TIA) is high at about one in six among stroke survivors, ${ }^{[6]}$ safe and effective secondary preventive measures can make an important impact on the burden of stroke in the community. Antiplatelet therapy reduces the risk of recurrent stroke by about a fifth ${ }^{[7]}$ and is now prescribed routinely. Other secondary preventive measures such as carotid endarterectomy ${ }^{[8]}$ and anticoagulant therapy. ${ }^{[9]}$ are suitable for only a small proportion of patients.

Angiotensin converting enzyme inhibitor (ACEI) and diuretic based prevention of recurrent stroke was demonstrated in the PROGRESS study ${ }^{[10]}$ and is recommended by international guidelines. ${ }^{[5]}$ In comparison to placebo, the ACEI perindopril ( $4.0 \mathrm{mg} /$ day) with or without indapamide (2.0-2.5 mg/day) reduced the risk of recurrent stroke by $28 \%$. The benefit was in addition to the protection provided by other antihypertensive agents and antiplatelet therapy.

This suggests the possibility of effectively using ACEI \pm indapamide-based treatment for prevention of recurrent stroke in day-to-day practice. However, there is no evidence that the risk reduction observed in the 
PROGRESS population under double blind randomized study conditions can be replicated in the primary care setting, especially in communities with different demographic and clinical characteristics.

The objective of this study was to assess the effect and acceptability of perindopril \pm indapamide based prevention of recurrent stroke, as reflected by its incidence, in the primary care setting of India.

\section{Materials and Methods}

A one step simple random sample procedure was used to select 13 cities in India. Physicians interested in stroke from these cities and the metropolitan cities of Mumbai, Calcutta, Delhi, and Chennai were identified by their membership of the Neurological Society of India. Of these, 39 neurologists, or physicians who managed stroke patients in their clinical practice agreed to participate in the study. A committee of the authors and investigators reviewed and accepted the study protocol.

In a prospective, non-comparative, open label study of 12 months duration during 2003-2004, participating physicians identified consecutive outpatients with a history of stroke ( 2 weeks after but within 5 years of the event) or TIA who were clinically stable for at least a fortnight and who were not receiving an ACEI, angiotensin receptor blocker or diuretic. Of these, patients without a specific indication or contraindication to an ACEI and diuretic or intolerance to these agents were selected. Stroke was defined as evidence of an acute disturbance of focal neurological function with symptoms lasting more than 24 hours and thought to be due to intracerebral hemorrhage or ischaemia. TIA was defined as evidence of an acute disturbance of focal neurological or monocular function with symptoms lasting less than 24 hours and thought to be due to arterioembolic or thrombotic vascular disease. Stroke was classified as ischaemic or hemorrhagic, on the evidence of either computed tomography or magnetic resonance imaging scan of the brain. Patients without neuro-imaging investigations were classified as having a stroke of uncertain type.

After giving their written informed consent, patients were assessed at baseline for demographic, clinical and treatment, characteristics shown in Table 1 . Sphygmomanometric blood pressure measurements were made under standard conditions ${ }^{[5]}$ and hypertension was defined as a previous record of at least two blood pressure readings above $140 / 90 \mathrm{mmHg}$ or the regular intake of antihypertensive drugs. Previous fasting plasma glucose greater than $126 \mathrm{mg} / \mathrm{dL}$ or the requirement of hypoglycemic drugs was used to diagnose diabetes. Hypercholesterolemia was identified as previous total plasma cholesterol of above $7.0 \mathrm{mmol} / \mathrm{L}$ or treatment with statins. Atrial fibrillation was recognized by positive history or ECG evidence. Coronary artery disease was diagnosed by previous known myocardial infarction or
Table 1: Baseline characteristics of outpatients with a history of stroke or transient ischaemic attack in the Indian primary care setting $\left(n=298^{*}\right)$

\begin{tabular}{llc}
\hline Age (years) & & $58.3 \pm 12.6$ \\
Male sex* & & $213(71.5)$ \\
Type of stroke or TIA & Ischaemic & $229(76.8)$ \\
& Haemorrhagic & $32(10.7)$ \\
& TIA & $31(10.4)$ \\
& unknown & $2(0.7)$ \\
Duration of stroke or TIA (months) & & $6.6 \pm 8.7$ \\
Association diseases & Hypertension & $231(77.5)$ \\
& Diabetes & $85(28.5)$ \\
& Dyslipidemia & $100(33.6)$ \\
& CAD & $60(20.1)$ \\
Under treatment for associated & Atrial fibrillation & $13(4.4)$ \\
diseases & Hypertension & $174(75.3)$ \\
& Diabetes & $63(74.1)$ \\
Prevention of recurrent stroke & Dyslipidemia & $81(81)$ \\
& Aspirin & $200(85.5)$ \\
& Antiplatelet & $137(58.8)$ \\
& Anticoagulants & $18(7.7)$ \\
Sytolic blood pressure (mmHg) & Endarterectomy & $3(1.3)$ \\
Diatolic blood pressure (mmHg) & & $154.0 \pm 23.9$ \\
\hline
\end{tabular}

${ }^{*} \pm$ value are means plus/minus standard deviation. All other values are numbers of patients with the percentages of the group in parenthesis; TIA = Transient ischaemic attack; $\mathrm{CAD}=$ coronary artery disease

stable angina according to the patient's medical record.

After baseline assessment, patients received the open label study medication and were followed up after 14, 90, 180, 270, and 365 days. For the first 14 days perindopril (COVERSYL, Serdia, India) was prescribed at a dose of $2 \mathrm{mg}$ per day. At subsequent follow ups, depending on blood pressure response and clinical judgment, physicians could adjust the study medication between perindopril 2, $4 \mathrm{mg}$ or a fixed dose combination of perindopril $4 \mathrm{mg}$ and indapamide $1.5 \mathrm{mg}$ (COVERSYL PLUS, Serdia, India). These study medications were taken once a day in the morning in addition to background medication for associated disease or other treatments for the secondary prevention of stroke. Patients purchased all drugs from the market on prescription.

At each follow up, patients were examined for the occurrence of acute cerebrovascular events, blood pressure level, compliance with study medication and side effects. Recurrent stroke or TIA was defined and classified as at baseline. The proportion of tablets ingested out of those prescribed, was used to assess compliance with medication. Side effects were evaluated by asking openended questions. The measurement of serum creatinine was repeated at the end of the study.

\section{Statistical analysis}

Data was analyzed on an intention to treat basis. The primary outcome variable was the annual incidence of non-fatal and fatal stroke. The Kaplan-Meier procedure was used to estimate the cumulative combined hazard of ischaemic stroke, hemorrhagic stroke, and TIA. Continuous variables were expressed as means with their standard deviation and categorical variables as percentages 
with related 95\% confidence intervals (CI).

\section{Results}

The 39 investigators recruited 298 outpatients with a stable stroke or TIA occurring between 2 weeks and 5 years after the event. The characteristics of these patients at baseline are summarized in Table 1. Most patients were males in their sixth decade, with an ischaemic stroke of 6 months duration. In about one in ten the cerebrovascular event was a hemorrhagic stroke or TIA. A majority of patients had mild to moderate hypertension, but about one in four were either not previously diagnosed or were not receiving antihypertensive treatment. One in four patients were normotensive. Nearly all patients were receiving antiplatelet agents or statins, which provide protection against the risk of recurrent stroke.

Patients were under observation for a mean duration of 327.1 (SD, 119.3) days. By the end of planned follow up (12 months), 47 (15.8\%) were lost to follow up, 15 (5.1\%) withdrew, of which 13 were due to causes unrelated to the study treatments. There were five $(1.7 \%)$ deaths during the study (1, due to cardiovascular; 3 , due to other; and 1 of indeterminate cause). At the end of study, the number (\%) of patients receiving the once daily study medications were 125 (41.9), perindopril 4 mg; 84 (28.2) fixed dose combination of perindopril and indapamide; and 22 (7.4) perindopril $2 \mathrm{mg}$. Mean compliance (\% of tablets ingested out of those prescribed) with study medications ranged from $98.1 \%$ at day 14 to $99.6 \%$ at the end of the study.

Overall, the mean decrease in systolic (95\% CI) / diastolic (95\% CI) blood pressure from baseline with perindopril \pm indapamide based treatment was 22.7 (19.8 to 25.6)/ 13.3 (11.7-14.9) $\mathrm{mmHg}$. The mean reduction in systolic/ diastolic blood pressure in hypertensive patients was 27.9 (24.7-31.1)/16.0 (14.4-17.6) $\mathrm{mmHg}$ and in normotensive patients, 2.4 (0-5.7)/2.85 (0.4-5.3) mmHg [Figure 1]. This decrease was maintained throughout the follow up, with no suggestion of attenuation in response.

During the 12 months of perindopril \pm indapamidebased treatment, the number (annual incidence \%) of recurrent cerebrovascular events were, ischaemic strokes 6 (2.0); hemorrhagic strokes, 2 (0.7); all strokes, 8 (2.7); TIA, 6 (2.0); and all strokes plus TIA 14 (4.7). There were no fatalities because of stroke. One patient developed stable angina and another suffered a myocardial infarction.

The Kaplan-Meier estimate of cumulative recurrent stroke or TIA hazard [Figure 2] at the end of one year's preventive treatment with the perindopril \pm indapamide based regimen was 3.3\% (95\% CI, 1.0-5.6).

Of the 16 (5.4\%) patients who reported side effects attributable to the study medication, two $(0.7 \%)$ withdrew from the study due to cough and 11 (3.7\%) patients complained of it at the first follow up after 14 days. At the end of 12 months treatment cough was

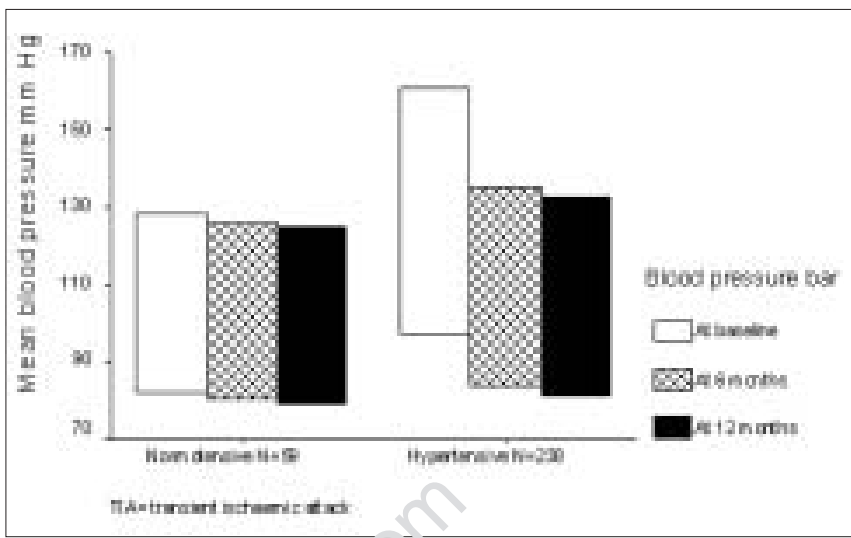

Figure 1: Effect of perindopril-based treatment on blood pressure in patients with stroke or TIA

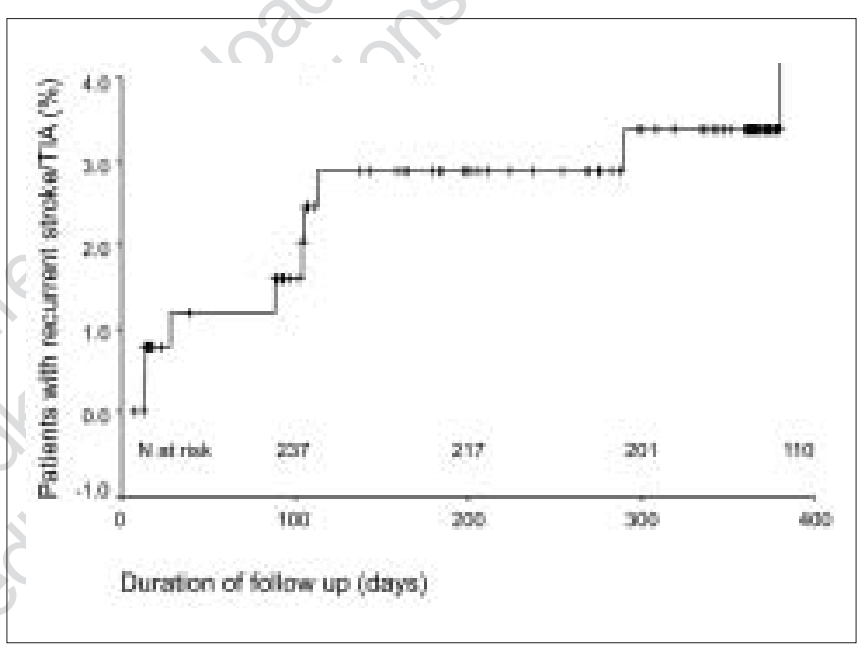

Figure 2: Cumulative hazard of recurrent stroke or TIA with perindopril-based treatment

a reported side effect in one patient. Other side effects were infrequent. One patient each complained of abdominal discomfort, nausea, feeling of weakness and giddiness.

\section{Discussion}

Patients with a previous stroke that was clinically stable or with a history of TIA, received a flexible blood pressure lowering regimen with perindopril alone or in combination with indapamide for 12 months. The treatment reduced blood pressure in hypertensive patients, with little effect on those who were normotensive. The annual recurrent stroke incidence while on perindopril \pm indapamide-based preventive treatment was $2.7 \%$ among patients at different stages of post stroke evolution ( 2 weeks to 54 months after their previous stroke or TIA) and against the background of other secondary preventive measures such as non-study antihypertensive drugs, antiplatelet agents, and statins. The treatment was acceptable to patients over the long term, with few side effects, a low frequency of cough and nearly complete compliance with the once daily dosage. 
Under the randomized double blind conditions of the PROGRESS study the annual recurrent stroke incidence in patients with a previous stroke or TIA assigned to the treatment arm was $2.7 \%{ }^{[10]}$ This study used similar recruitment criteria and active drug treatments as PROGRESS. However, our patients were about 6 years younger. Another major difference was a nearly $30 \%$ greater frequency of hypertension, that was about 10 mmHg higher at baseline in comparison to patients recruited in PROGRESS. Perhaps this difference in baseline blood pressure partly explains the large reduction of $22.7 / 13.3 \mathrm{mmHg}$ in blood pressure seen in this study compared to the more modest $9 / 4 \mathrm{mmHg}$ decrease in PROGRESS. Another reason could be the greater efficiency of both ACEI and diuretics in younger patients because of their relatively higher renin status. Despite these population differences, the incidence rate of recurrent stroke observed in the Indian primary care setting was similar. This suggests that perindopril \pm indapamide based prevention of recurrent stroke may be equally effective in populations with a different demographic and blood pressure profile than in PROGRESS.

The lack of a randomized double blind study design to compare the treatment with other blood pressure lowering medication or placebo is a study limitation and the results do not directly demonstrate that perindopril \pm indapamide-based treatment reduces the risk of recurrent stroke. However, the objective was to assess the effect and acceptability of perindopril with or without indapamide-based prevention of recurrent stroke, as reflected by its incidence, in the primary care setting of India. A randomized protocol, involving stricter organization and follow up, may not have been reflective of primary care practice conditions. By contrast, this study was on outpatients of randomly selected physicians distributed throughout India. The study treatments were prescribed and purchased by patients from the market and follow up was under normal practice conditions. Other study limitations that could influence the results were a low stroke/TIA event rate due to the small sample size and relatively short duration of follow up. The proportion of patients lost to follow up was high and this could potentially under-estimate the primary outcome.

In the context of Indian primary care, patients with a previous stable stroke or TIA are younger and have a higher degree of blood pressure, which is also more frequent in comparison to Western and other Eastern patients. In these patients, treatment with a flexible perindopril \pm indapamide based regimen, in addition to other antihypertensive and secondary preventive medication was associated with an annual recurrent stroke incidence similar to that observed under double blind randomized conditions. The result suggest that perindopril \pm indapamide based prevention effectively reduces the risk of recurrent stroke in the setting of day to day clinical practice and among younger patients with higher levels of blood pressure. However, these results need to be confirmed in controlled studies of longer duration on a larger number of patients.

\section{Acknowledgements}

We are indebted to the following investigators: Dr. Singh MM (Agra); Dr. Acharya R, Dr. Joshi N (Ahmedabad); Dr. Agadi JB, Dr. Mruthyunjayanna BP, Dr. Roy AK, Dr. Srinivasa R (Bangalore); Dr. Agarwal VK (Bareilly); Dr. Asokan K (Coimbatore); Dr. Achtani R, Dr. Dhamija RM, Dr. Garg R, Dr. Goyal V, Dr. Mehndiratta MM, Dr. Sairam N (Delhi); Dr. Lowalekar KB (Gwalior); Dr. Prasad VS, Dr. Reddy PG (Hyderabad); Dr. Sharma D (Jaipur); Dr. Gajre S (Jalgaon); Dr. Chatterjee A, Dr. Das A, Dr. Nandi SS, Dr. Roy T (Kolkata); Dr. Agarwal A, Dr. Garg RK, Dr. Roy AK, Dr. Shakil M (Lucknow); Dr. Kini G, Dr: Surya N (Mumbai); Dr. Motiani R (Noida); Dr. Dhairyawan P, Dr. Satyanarayana G (Secunderabad); Dr. Prabhakaran S (Trivandrum); Dr. Prabhakararao Y (Vishakhapatnam). The study was supported by a financial grant from Serdia Pharmaceuticals (India) Pvt. Ltd., the manufacturers of perindopril and its fixed dose combination with indapamide to cover organizational expenses. No other financial consideration was involyed.

\section{References}

1. WHO. World Health Report 1999. WHO: Geneva; 1999,

2. Hankey G, Warlow C. Treatment and secondary prevention of stroke: Evidence, costs and effects on individuals and populations. Lancet 1999;354:1457-63.

Anand K, Chowdhury D, Singh KB, Pandav CS, Kapoor SK. Estimation of mortality and morbidity due to strokes in India. Neuroepidemiology 2001;20:208-11.

4. Caplan LR. Treatment of Acute Stroke still struggling. JAMA 2004;292:1883-5

5. Chobanian AV, Bakris GL, Black HR, Cushman WC, Green LA, Izzo JL Jr, et al. The Seventh Report of the Joint National Committee on Prevention, Detection, Evaluation, and Treatment of High Blood Pressure: The JNC 7 report. JAMA 2003;289:2560-72.

6. Hankey GJ, Warlow CP. Treatment and secondary prevention of stroke: Evidence, costs and effects on individuals and populations. Lancet 1999;354:1457-63

7. Algra A, Van Gijn J. Cumulative meta-analysis of aspirin efficacy after cerebral ischaemia of arterial origin. J Neurol Neurosurg Psychiatry 1999;66:255.

8. European Carotid Surgery Trialists' Collaborative Group. Randomised trial of endarterectomy for recently symptomatic carotid stenosis; final results of the MRC European Carotid Surgery Trial (ECST). Lancet 1998;351:1379-87.

9. Hart RG, Benavente O, McBride R, Pearce LA. Antithrombotic therapy to prevent stroke in patients with atrial fibrillation: A meta-analysis. Ann Intern Med 1999;131:492-501.

10. Progress Collaborative Group Randomized trial of a perindoprilbased blood-pressure-lowering regimen among 6105 individuals with previous stroke or transient ischaemic attack Lancet 2001;358: $1033-41$

Accepted on 26-12-2006

Source of Support: Grant from Serdia Pharmaceuticals (India) Pvt. Ltd., Conflict of Interest: None declared. 